\title{
Oriental Thinking and Fuzzy Logic, Celebration of the 50th Anniversary of Fuzzy Sets
}

\author{
Peizhuang Wang ${ }^{1}$
}

Published online: 2 January 2016

(C) Springer-Verlag Berlin Heidelberg 2016

\section{Preface}

In August 17-20, 2015, during the spring up of big data tide, we have held an international conference on Oriental Thinking and Fuzzy Logic at Dalian, China to celebrate the 50th anniversary of Fuzzy Sets. The honorary chair for this conference was, of course, the founder of fuzzy sets theory, Prof. L. A. Zadeh. All colleagues at the conference expressed their deep respects to him. Every Chinese scholar who has been met him remembers by his modesty and enthusiasm. To guide the information revolution, he has constructed a great bridge between qualitative and quantitative.

There were five main topics in the conference: Fuzzy information processing and engineering; Internet and big data applications; Factor space and factorial neural networks; Information granulation and granular computing; Extenics and innovation methods. Here, Extenics is a new field of the disciplinary, which was initiated by Prof. Wen Cai to achieve innovation facing a problem seems impossible to be possible. The theory of factor space was initiated by me with the oriental thinking.

There were 15 plenary talks in the conference as follows: Wen Cai, Fuzzy logic and Extenics; Yixiang Chen, Inter-definability and application of fuzzy logic operators; I. Dzitac, Fuzzy logic and artificial intelligence; Jiali Feng, Theory of meta-synthetic wisdom based on fusion of qualitative, quantitative and imagery operations; Jiafa Gu, System science and Chinese medicine; He Ouyang, A mathematical foundation for factor spaces; Qing He, Uncertainty Learning; Congfu Huang, An approach checking whether an intelligent internet can improve intelligence; Deyi Li, Cognitive physics; Zengliang Liu, Factorial neural networks; W. Pedrycz, New frontiers of computing

\footnotetext{
Peizhuang Wang peizhuangw@126.com

1 Beijing, China
} 
and reasoning with qualitative information: A perspective of granular computing; Germano Resconi, From inconsistent topology to consistent in big data; Yong Shi and Yingjie Tian, Uncertainty and big databases; Peizhuang Wang, Fuzzy sets and factor space; Zeshui Xu, Complex information decision making. As a special guest, Mr. Haoran Lin introduces his high school book "Preliminary of Fuzzy Mathematics" and 18 years teaching practice of him in Shanghai High School.

Apart from the organized speeches, we are more appreciated by articles come from individuals with natural interesting and deep friendship toward Prof. L. A. Zadeh. They developed fuzzy theory along probability representation, rough sets, intuitionistic fuzzy sets, nonlinear PSO, structure elements ranking method and they apply fuzzy theory into recommendation, feature extraction, qualitative mapping etc. Among all papers presented at the conference, we have carefully selected eight papers to form this special issue as assorted appetizers to commemorate the 50th anniversary of fuzzy sets from Dalian conference.

As the chair of Advisory Committee, I would like to thank for the valuable contributors: Professors Wen Cai, F. G. Filip, Ioan Dzitac, Guirong Guo, jifa Gu, Xingui He, Deyi Li, Wei Li, Yingming Liu, M. Mizumoto, Jinping Ou, W. Pedrycz, Germano Resconi, R. Slowinski, Hao Wang, Shouyang Wang, Congxin Wu, Wangmin Wu, Weixin Xie, Zongben Xu, T. Yamakawa, Bingru Yang, Jianying Zhu, Wujia Zhu, and Zhaowen Zhuang. I also thank the members in the organizing institute under chairs Professors Jiren Wang and Kaiqi Zou as well as the members in the program committee under Chair Professor Yong Shi. I deeply appreciate the leadership of the conference chair Professor Zengliang Liu. Finally, we thank the managing editor of AODS, Prof. Yingjie Tian who made this special issue possible! 